

\title{
POST-OPTIMALITY ANALYSIS IN AEROSPACE VEHICLE DESIGN
}

\author{
Robert D. Braun* \\ NASA Langley Research Center \\ Hampton, VA \\ Ilan M. Kroo** \\ Peter J. Gage*** \\ Stanford University \\ Stanford, CA
}

\begin{abstract}
This analysis pertains to the applicability of optimal sensitivity information to aerospace vehicle design. An optimal sensitivity (or post-optimality) analysis refers to computations performed once the initial optimization problem is solved. These computations may be used to characterize the design space about the present solution and infer changes in this solution as a result of constraint or parameter variations, without re-optimizing the entire system. The present analysis demonstrates that post-optimality information generated through first-prder computations can be used to accurately predict the effect of constraint and parameter perturbations on the optimal solution. This assessment is based on the solution of an aircraft design problem in which the post-optimality estimates are shown to be within a few percent of the true solution over the practical range of constraint and parameter variations. Through solution of a reusable, single-stage-to-orbit, launch vehicle design problem, this optimal sensitivity information is also shown to improve the efficiency of the design process. For a hierarchically decomposed problem, this computational efficiency is realized by estimating the main-problem objective gradient through optimal sensitivity calculations. By reducing the need for finite differentiation of a re-optimized subproblem, a significant decrease in the number of objective function evaluations required to reach the optimal solution is obtained.
\end{abstract}

\section{Nomenclature}
A active constraint Jacobian, dimension $\mathbf{m} \times \mathbf{n}$
AR aspect ratio
c active constraint vector, dimension $\mathbf{m}$

*Aerospace Engineer, Space Systems Division, Member AlAA. **Associate Professor, Aeronautics \& Astronautics, Member ALAA.

***Research Assistant, Aeronautics \& Astronautics, Student Member AIAA,

Copyright 1992 American Institute of Aeronautics and Astronautics, Inc. No copyright is asserted in the United States under Title 17,U.S. Code. The U.S. Government has a royalty-free license to exercise all rights under the copyright claimed herein for Governmental purposes. All other rights are reserved by the copyright owner.

$\begin{array}{ll}\mathrm{C}_{\mathrm{L}} & \text { surface lift coefficient (wing or tail) } \\ \text { CONSIZ } & \text { Configuration Sizing program } \\ \text { DOC } & \text { direct operating cost, cents/seat-mile } \\ \text { DOF } & \begin{array}{l}\text { degree-of-freedom } \\ \text { objective function }\end{array} \\ \text { F } & \text { objective gradient vector, dimension n } \\ \mathbf{g} & \text { gross liftoff weight } \\ \text { GLOW } & \text { number of parameters } \\ \text { LaRC } & \text { Langley Research Center } \\ \text { m } & \text { number of active constraints } \\ \text { MR } & \text { mass-ratio } \\ \text { n } & \text { number of design variables } \\ \text { NPSOL. } & \text { Nonlinear Programming, Stanford } \\ & \text { Optimization Laboratory } \\ \text { p } & \text { parameter vector, dimension } k \\ \text { PASS } & \text { Program for Aircraft Synthesis Studies } \\ \text { POST } & \text { Program to Optimize Simulated Trajectorie } \\ \text { Sref } & \text { reference aerodynamic surface area, } \mathrm{ft}^{2} \\ \text { SSTO } & \text { single-stage-to-orbit launch vehiclo } \\ \text { U/c } & \text { thickness-to-chord ratio } \\ \text { T/W } & \text { thrust-to-weight ratio } \\ \text { x } & \text { design variable vector, dimension } n \\ \text { Ac } & \text { constraint value perturbation } \\ \lambda & \text { Lagrange multiplier vector, dimension m }\end{array}$

superscript

* evaluated at the optimum solution subscript

c computed variable

Intraduction and Background

Aerospace vehicle design is an iterative process which requires the integration of numerous disciplinary analyses (e.g., aerodynamics, structures, propulsion, performance, and cost). Often, the time required to set-up the design problem. model the disciplinary interactions, and obtain an optimum solution is significant. Therefore, once a solution is reached. it is imperative to extract as much design information *s 
possible. In addition to design variable information pertaining to the optimum, it is possible to obtain a description of the design space about the optimal configuration through a post-optimality (or sensitivity) analysis. In particular, an optimal sensitivity analysis may be used to infer the change in the present optimal design with respect to a small change in a constraint or a previously fixed parameter. This information which is generally available without having to reoptimize the entire system may also be advantageously used in the solution of a decomposed optimization problem. The present investigation focuses on the application of optimal sensitivity analyses to aerospace vehicle design.

Sensitivities are typically used in design of complex systems to compute the change in the set of output variables (including the constraints and objective function) to a small change in a given design variable (while holding the other design variables fixed). 1,2 When coupled with an optimization scheme, the sensitivity information is used to move the design variables in the direction of the optimal solution. Additionally, the sensitivity calculations may be used to extract information regarding the disciplinary couplings within a complex system. Because this application of sensitivities is used to improve a design configuration, this approach may be referred to as a design sensitivity analysis. For example, in aircraft design, design sensitivity information can be used anywhere in the design space to infer how a change in the wing aspect ratio, sweep, or twist affects the aircraft's overall weight, or direct operating cost (DOC) without violating a range constraint. Additionally, through a design sensitivity analysis, the coupling between structures and aerodynamics or weights and performance can be assessed.

In a preliminary design environment, the solution of a single optimization problem is seldom satisfactory. In addition to the present solution, the impact of design changes on the optimal configuration is of significant interest. Fortunately, this information is generally available without having to re-optimize the entire system. ${ }^{3-11}$ The use of sensitivities in this fashion (where the relationships involve optimal variables) may be termed an optimal sensitivity analysis. In terms of the aircraft design problem discussed previously, utilizing sensitivity information at an optimal design point, one can infer how a small change in the range constraint would change the optimum DOC and optimum design variables (wing aspect ratio, sweep, and twist).

An optimal sensitivity (or post-optimality) analysis can take on many forms, each providing a different level of in- formation concerning changes in the optimal solution. The simplest form of post-optimality analysis requires only an accurate prediction of the Lagrange multipliers at the solution. These multipliers may be used to infer changes in the optimum solution with respect to small changes in an active constraint and are generally provided upon solution to an optimization problem with little or no additional computational requirements. For a small increase in computational effort, the change in the optimum solution with respect to a change in a previously fixed parameter may be attained. This is the level to which post-optimality information is used in the present investigation; however, with more numerical effort, the altered set of optimum design variables may also be obtained with respect to a small change in either an active constraint or previously fixed parameter.

The present study begins with a brief discussion of the first-order computational approach used to compute the optimal sensitivity information. The validity of these estimates is then discussed through solution of two optimization problems: (1) Rosenbrock's valley function and (2) the design of a commercial transport aircraft. Through these applications, the utility and limitations of post-optimality information is demonstrated. Of specific interest, is the determination of whether typical aerospace design constraints and parameters are well-suited to a first-order post-optimality analysis. Comparisons between the optimal sensitivity predictions and re-optimization are also presented. With the validity of these post-optimality estimates established, the sensitivity information is used to improve the efficiency of the design process without loss of accuracy. In particular, the computational advantage of using optimal sensitivity information in a hierarchically-decomposed, single-stage-to-orbit (SSTO), launch vehicle design problem is demonstrated.

\section{Analysis}

\section{Methodology}

There are several available methods for computing optimal sensitivity information. These techniques are summarized in References 3, 5, 8 and 9. In general terms, a nonlinear programming problem may be mathematically expressed in terms of the design variables $\left(x_{\mathfrak{i}}\right)$, problem constraints $\left(c_{\mathfrak{i}}\right)$, and an objective function $(F)$ as

$$
\begin{array}{ll}
\text { minimize } F, \text { where } & F=F\left(x_{1}, x_{2}, \ldots . . x_{n}\right) \\
\text { subject to } & c_{i}=c_{i}\left(x_{1}, x_{2}, \ldots . . x_{n}\right), i=1, m
\end{array}
$$


This problem will also be characterized by a number of parameters $\left(p_{j}, j=1, k\right)$ which remain fixed during the optimization procedure. For an aircraft, these parameters may include the cruise Mach number, the maximum cruise range, or the number of passengers. For a launch vehicle such parameters could include engine propulsion characteristics, payload, weight margin, or tankage weight fractions. Once the optimization problem is solved, the change in the optimal objective function. with respect to any fixed parameter may be calculated by

$$
\frac{d F^{*}}{d p}=\frac{\partial F}{\partial p}+\lambda_{i}^{T^{*}} \frac{\partial c_{i}}{\partial p}
$$

Here $\lambda *$ represents the Lagrange multiplier vector at the solution. Note that if the parameter of interest is an active constraint bound, this equation reduces to

$$
\frac{\mathrm{dF}^{*}}{\mathrm{dc}}=\lambda^{*}
$$

Most optimization algorithms provide an estimate of $\lambda$ * at the solution. However, in finite precision, differences among the various techniques may be significant. At the solution of the problem, the necessary and sufficient conditions of optimality yield

$$
A^{T} \lambda^{*}=g
$$

The solution to eq. (3) may be obtained by solving the equivalent linear system

$$
\left(\mathrm{AA}^{\mathrm{T}}\right) \lambda^{*}=\mathrm{Ag}
$$

however, such a solution is subject to potential conditioning problems as the condition number of $\mathrm{AA}^{\mathrm{T}}$ is the square of the condition number of $A$. In finite precision, a more accurate technique for obtaining $\lambda^{*}$ is by forming either a TQ factorization $^{4}$ or a $Q R$ decomposition 7,11 of $A$.

\section{Limitations and Operational Cost}

With the above analysis, the change in optimal solution with respect to a change in a given constraint or design parameter may be estimated. However, because this estimate is only valid in a region about the optimum where changes are occurring linearly, we may be limited to small perturbations in the parameters. Additionally, the active set of constraints must not be altered by the perturbation. To illustrate the potential problems induced by an active set change, con- sider the simple case of a single active constraint which is initially close to the minimum unconstrained solution. If this constraint is perturbed past the unconstrained optimum (making the constraint inactive), it is clear that the optimal objective function would be the global minimum. However, the sensitivity estimate (having no means to compute the unconstrained minimum) would predict a linear variation in the objective function continuing to values well below the global minimum.

Other considerations which effect the valid extrapolation range of the sensitivity estimates include problem scaling and convergence tolerance. For a problem with numerous active constraints or numerous parameters of interest, scaling is always a general optimization concern. In particular, Refs. 7 and 11 demonstrate that the Lagrange multiplier estimates calculated in the scaled space and then transformed back to the unscaled design space are generally more accurate than $\lambda^{*}$ estimates computed directly in the unscaled space. Additionally, although many optimizers provide an estimate of $\lambda^{*}$ as part of the termination process, accurate estimates are only ensured when the problem has converged tightly.

For an optimization routine that does not already provide an estimate of $\lambda^{*}$, the operational cost of adding such an analysis is quite small relative to the cost of the optimization itself. In fact, at the solution, a general quadratic optimization routine may already provide an estimate of $g$ and have performed the required factorization of $A$. If a TQ factorization of $A$ has been performed, the additional computations needed to produce $\lambda *$ require on the order of $\left(n m+m^{2}\right)$ operations. Note that this process does not require any additional function or gradient evaluations. With accurate estimates of $\lambda^{*}$, the change in optimal objective function with respect to a constraint variation is known. However, to obtain a prediction of the change in optimal objective function with respect to a parameter variation, one or two extra function evaluations (depending on the choice of finite-differencing approximation) are required to solve eq. (1) for each parameter of interest.

\section{Results and Discussion}

\section{Rosenbrock's Valley Function}

Since the optimal sensitivity predictions are in effect a first-order approximation, this information is only expected to be valid in a small region about the optimum. The size of this region is dependent on the linearity of the design space 
in the direction orthogonal to the constraint. To illustrate this regional dependence, minimization of Rosenbrock's twovariable valley function was performed with a single linear constraint. Rosenbrock's valley function is

$$
F\left(x_{1}, x_{2}\right)=100.0\left(x_{2}-x_{1}{ }^{2}\right)^{2}+\left(1.0-x_{1}\right)^{2}
$$

This design space is displayed in Fig. 1, where the contours shown represent constant values of the objective function. As shown in this figure, application of the optimal sensitivity estimates is performed in both a linear and nonlinear region of the design space. The optimal sensitivity value was computed and compared with the actual change in optimal objective function $\left(\mathrm{F}^{*}\right)$ obtained through reoptimization. Optimization was performed with the sequential quadratic programming algorithm, NPSOL, which uses a quasi-Newton method to approximate the Hessian. ${ }^{12}$ At the solution, NPSOL provides an estimate of $\lambda^{*}$ (obtained through a TQ factorization of $A$ in the scaled design space). To eliminate finite difference effects, the objective-gradient, Jacobian, and Hessian were computed analytically for this sample problem.

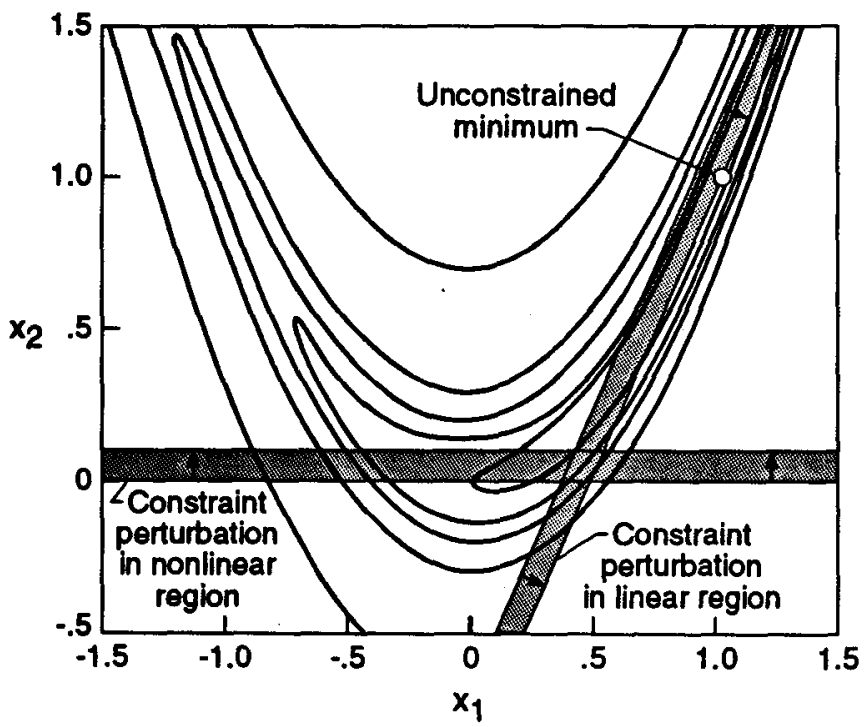

Fig. 1 Rosenbrock's valley function.

Comparison between the sensitivity estimate and the actual change in optimal solution were made for various changes in the constraint and are illustrated in Figs. 2 and 3. As shown in Fig. 2, for extremely small changes in the constraint over a highly nonlinear region of the design space, the sensitivity prediction agrees well with the actual change. However, for perturbations in the constraint value $(\Delta c)$ greater than approximately $5 \%$, the linear approximation begins to break down. For larger changes, the sensitivity prediction is highly inaccurate and its application leads to an erroneous result. On the other hand, for the design region and constraint of Fig. 3, the sensitivity prediction agrees quite well over most of the design space. Note that in this case the unconstrained minimum is reached for $a \Delta c$ of 0.173 $\left(F^{*}=0\right)$. With a larger variation in the value of the constraint, the optimal sensitivity estimate continues to predict a decrease in the objective function when in fact one cannot occur. This illustrates the change in active-set problem discussed earlier. The good agreement between the sensitivity prediction and actwal change in $\mathrm{F}^{*}$ shown in Fig. 3 is a result of the linearity of this region of the design space in the direction of the constraint variation. Hence, both the topography of the design space and direction of the constraint are significant in determining the validity of the optimal sensitivity prediction.

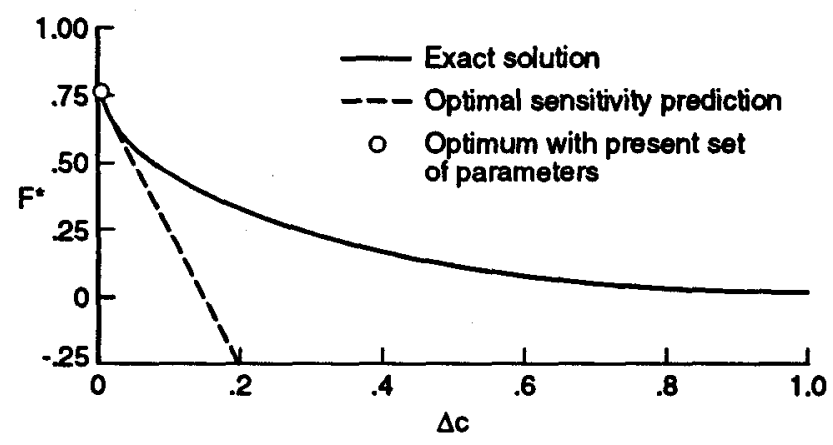

Fig. 2 Extrapolation and validity of optimal sensitivity prediction in nonlinear region of the design space.

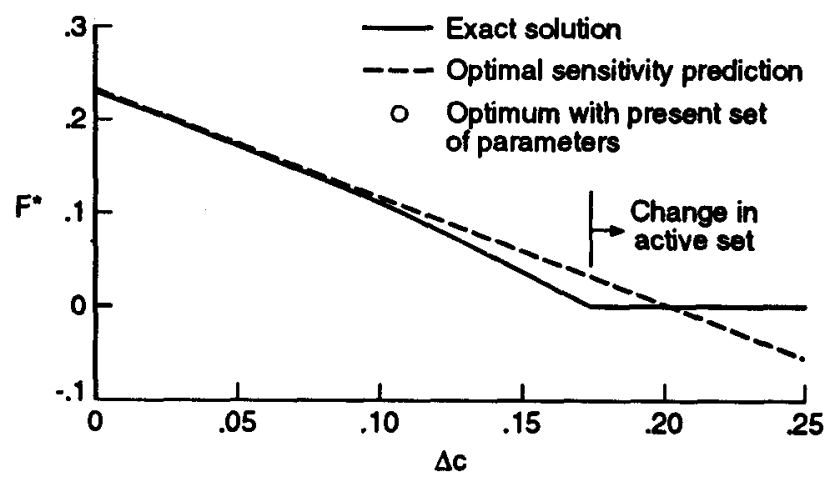

Fig. 3 Extrapolation and validity of optimal sensitivity prediction in linear region of the design space.

\section{Optimal Sensitivity Analysis in Aircraft Design}

To illustrate the applicability of optimal sensitivity analysis to a more complex, aerospace design problem, a DC-9 class, commercial transport aircraft was analyzed with the use of the PASS system. ${ }^{13-16}$ PASS is a quasi-procedural 
aircraft design system consisting of numerous disciplinary analysis and optimization routines. The aircraft design problem is posed with twelve design variables, and nine constraints. As listed in Table 1, the constraints include limits on static stability, landing and takeoff field lengths, cruise range and thrust, and climb-gradient in the event of an engine failure. Design variables include the initial and final cruise altitude, the wing and horizontal-tail geometrical properties, the uninstalled engine thrust level, and the flap setting at takeoff. Note that two additional constraints and design variables are included to satisfy compatibility requirements between the analysis routines, thereby eliminating an iteration loop. ${ }^{16}$ Use of compatibility constraints is discussed in the next section of this paper. The analysis routines used to solve this problem are based on those used in Ref. 13 with some modification. NPSOL ${ }^{12}$ was utilized to minimize direct operating cost.

Table 1. Aircraft design problem: DC-9 class, commercial transport.

Objective function = direct operating cost

Constraints

\begin{tabular}{|c|c|}
\hline Name & Max. \\
\hline 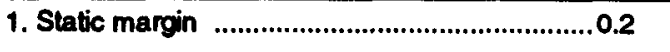 & 1.0 \\
\hline 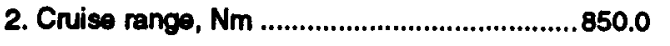 & 960.0 \\
\hline 3. (Drag/thrust) at cruise ........................................ 0.3 & 0.88 \\
\hline 4. Second-segment climb gradient .....................0.024 & 0.030 \\
\hline 5. $C_{L}$ of vertical tail with engine out .......................... & 0.8 \\
\hline 6. Takeoff field length, ft .....................................5000.0 & 7500.0 \\
\hline 7. Landing field length, ft ......................................2000.0 & 6000.0 \\
\hline 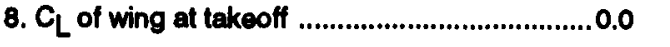 & 2.39 \\
\hline 9. $C_{L}$ of horizontal tail at takeoff ............................. 0.0 & 0.8 \\
\hline
\end{tabular}

Design Variables

\begin{tabular}{|c|c|c|}
\hline Name & Initial Value & Max. \\
\hline 1. Initial cruise altitude, ft ................. 10000.0 & 31000.0 & 50000.0 \\
\hline 2. Final cruiso altitude, $\mathrm{ft} \ldots . . . . . . . . . . .10000 .0$ & 31000.0 & 50000.0 \\
\hline 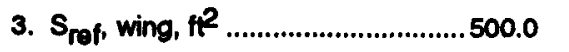 & 1000.7 & 1500.0 \\
\hline 4. AR, wing & 8.7 & 12.0 \\
\hline 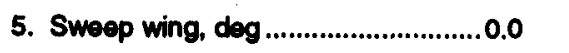 & 24.5 & 50.0 \\
\hline 6. $v_{c}$, wing & 0.1123 & 0.16 \\
\hline 7. Wing longitudinal position .................. & 0.35 & 0.45 \\
\hline 8. $S_{\text {ref }}$, horizontal tail ............................75.0 & 250.18 & 450.0 \\
\hline 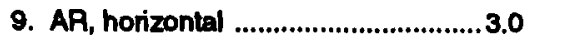 & 4.928 & 10.0 \\
\hline 10. Engine static thrust level, lb ....... 10000.0 & 14000.0 & 20000.0 \\
\hline 11. Maximum zoro fuel weight, ib ....30 000.0 & 87650.0 & 9000.0 \\
\hline 12. Takeoff flap setting, deg .................. 0.0 & 5.0 & 50.0 \\
\hline
\end{tabular}

From the starting point listed in Table 1, 27 major iterations or 610 objective function evaluations were required for NPSOL to converge to the optimum solution. The values of the optimum design variables and objective function are presented in Figure 4 along with a vehicle schematic. As part of the PASS analysis, the scaled Lagrange multiplier estimates (calculated at the solution for each active constraint by NPSOL) are transformed to the unscaled design space. These unscaled multiplier estimates are listed in Table 2 along with the value of each active constraint at the solution. At the solution there are six active constraints, not including the two compatibility relations. The final column in Table 2 presents $c \lambda *$, the value of the constraint at the optimum times the corresponding Lagrange multiplier estimate. This value may be used as a guideline in comparing the effect of constraint variations of different units. Comparing the $c \lambda^{*}$ values listed in Table 2, it is clear that a small in-

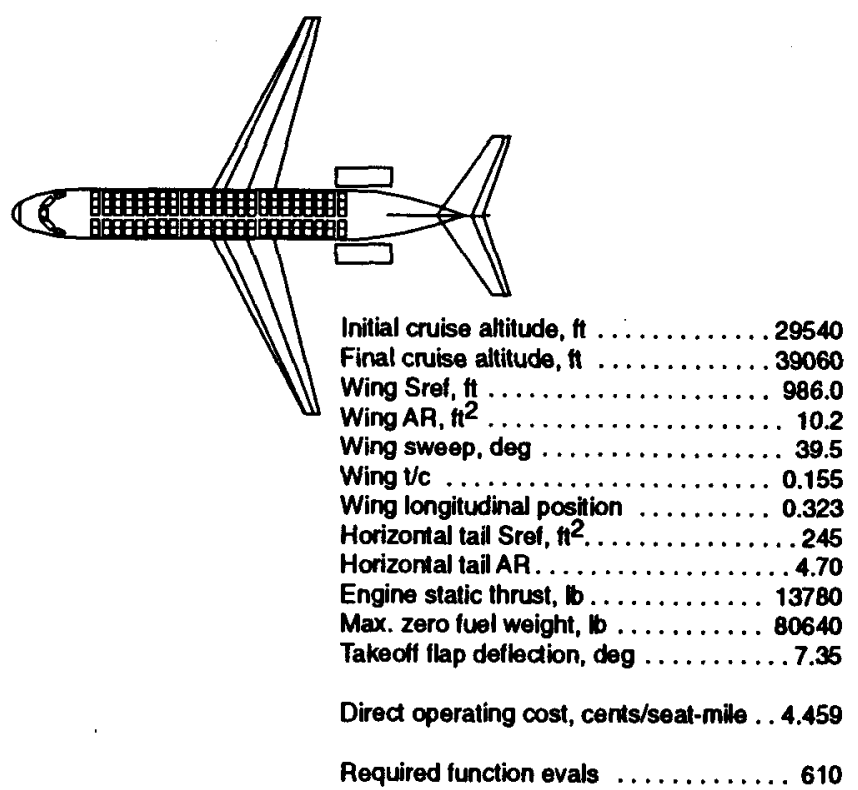

Fig. 4 Optimal solution of commercial transport aircraft design problem.

Table 2. Aircraft design problem: active constraints and Lagrange multiplier estimates.

\begin{tabular}{|l|c|c|c|}
\hline \multicolumn{1}{|c|}{ Active Constraints } & Value, $\mathrm{c}$ & $\lambda^{*}$ (unscaled) & $\mathrm{c} \lambda^{*}$ \\
\hline Static margin & 0.2 & $1.0170 \theta-01$ & 0.0234 \\
Cruise range, Nm & 960.0 & $-9.7517 \theta-04$ & -0.9360 \\
(Drag/thrust) at cruise & 0.88 & $-3.1136 \theta-02$ & -0.0274 \\
Second-segment climb gradient & 0.024 & 7.0939 & 0.1703 \\
$C_{L}$ of wing at takeoff & 2.39 & $-6.7056 \theta-02$ & -0.1602 \\
Takeoff field length, ft & 7500.0 & $-6.6704 \theta-05$ & -0.5003 \\
\hline
\end{tabular}


crease in cruise range is most beneficial toward minimizing DOC*. A small increase in takeoff field length is next in significance followed by either a decrease in the secondsegment climb requirement or an increase in the allowable wing lift-coefficient at takeoff.

Comparisons between the optimal sensitivity prediction and the actual change in optimal solution were made for various changes in each active constraint. These comparisons are illustrated in Figures 5-9. As is clear from each figure, the optimal sensitivity estimate is nearly exact in the limit of an infinitesimal variation of each constraint value. Surprisingly, the estimates hold up rather well over a relatively large range of constraint values. For example, as depicted in Fig. 5, for cruise range constraint variations on the order of $100 \mathrm{~nm}$, the optimal sensitivity estimate provides a good prediction of the change in DOC* (to within 1.5\%). Clearly, if the cruise range is perturbed 500 to $1000 \mathrm{~nm}$, the optimal sensitivity prediction becomes invalid. This results from both the nonlinearity of the design space and changes

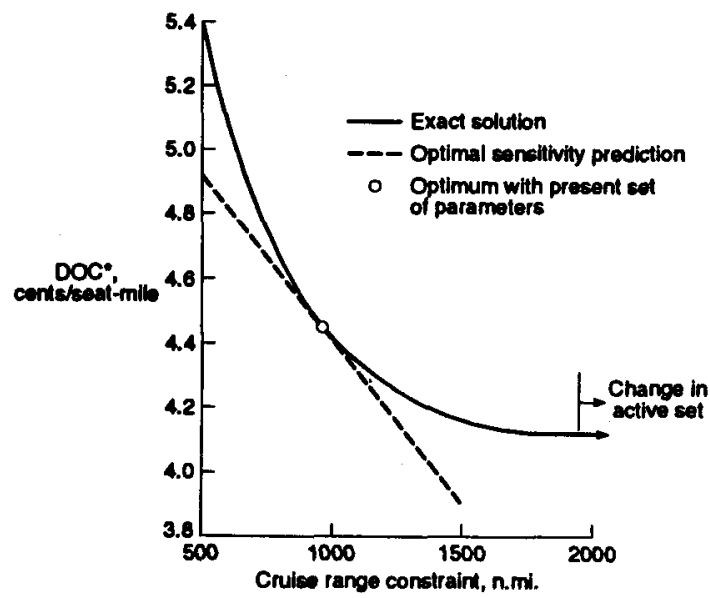

Fig. 5 Comparison of optimal sensitivity prediction for cruise range constant variations.

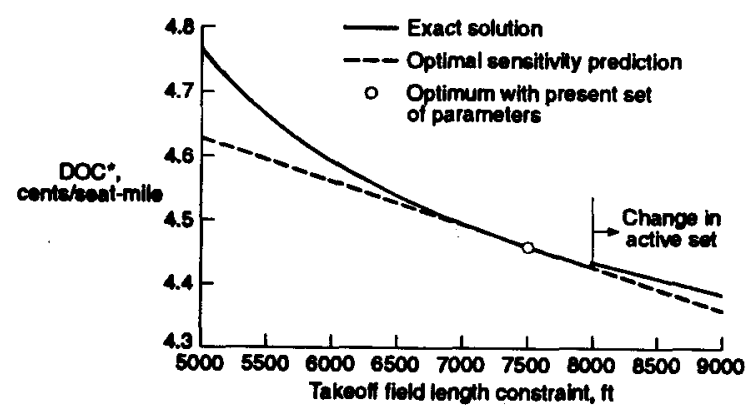

Fig. 6 Comparison of optimal sensitivity prediction for takeoff field length constraint variations.

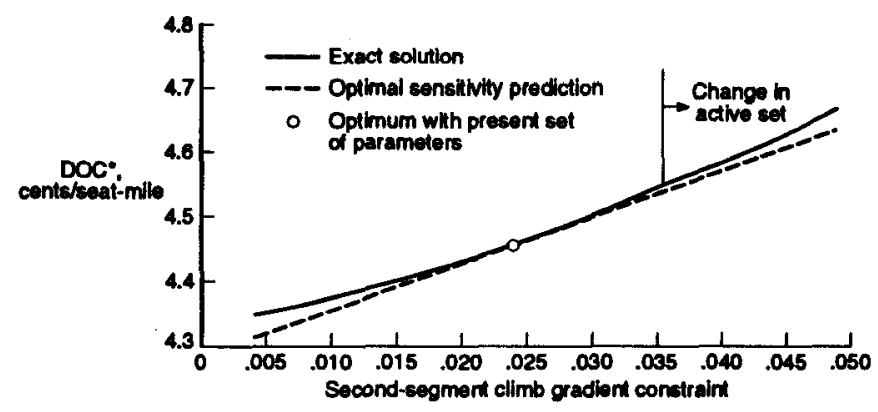

Fig. 7 Comparison of optimal sensitivity prediction for second-segment climb gradient constraint variations.

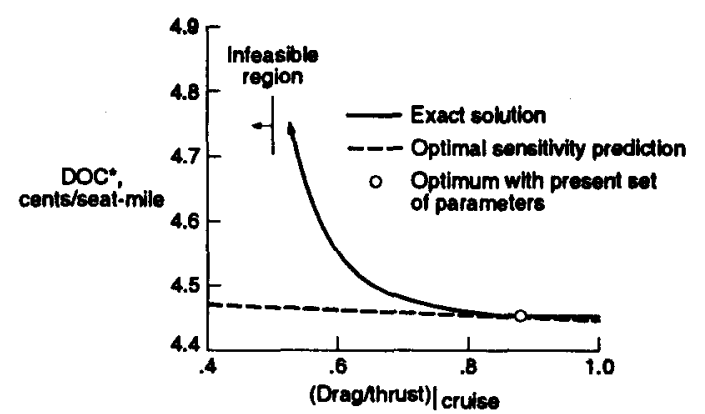

Fig. 8 Comparison of optimal sensitivity prediction for ratio constraint variations.

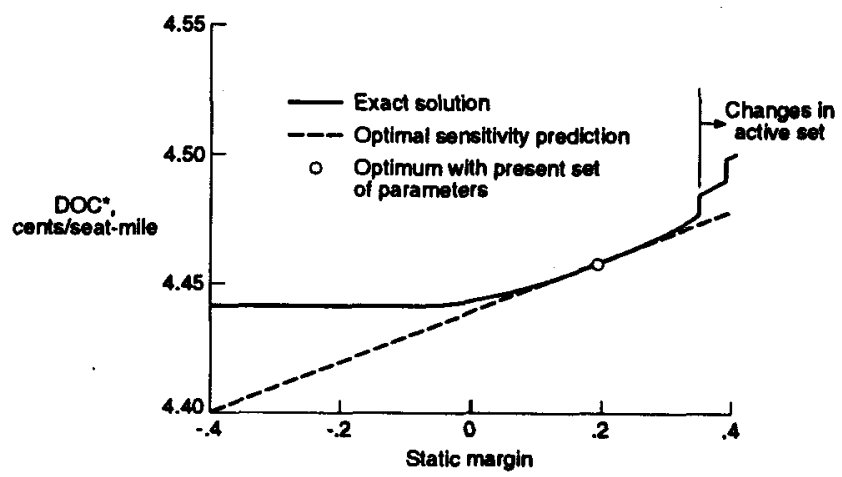

Fig. 9 Comparison of optimal sensitivity prediction for static margin constraint variations.

in the active set of constraints. For instance, beyond a constraint value of $1951 \mathrm{~nm}$, the cruise range constraint becomes inactive and further increases in this variable do not result in a lower DOC*, as predicted. However, for constraint perturbations of this magnitude, we are really dealing with a different design problem rather than a perturbation of the original problem; hence, one would not expect these estimates to be valid. 
Figures 6 and 7 depict analogous comparisons in regard to takeoff field length and second-segment climb gradient constraint perturbations. These figures show that throughout the entire practical range of interest, the linear sensitivity estimates predict the change in DOC* quite well (within $1 \%$ for second-segment climb gradient variations and within 3\% for takeoff field length perturbations). Surprisingly, these post-optimality predictions are still found to be accurate after a change in the active set of constraints.

Figures 8 and 9 show the change in optimum DOC with respect to changes in the cruise drag-to-thrust ratio and static margin constraints. Once again, note that in the vicinity of the present solution (static margin range of $0.1-0.3$ and cruise drag-to-thrust ratio from 0.7 to 1.0 ), the agreement is quite good (within 1\%). However, these figures also show the potential danger of extrapolating beyond the prediction's linear region of validity. For the case of cruise drag-to-thrust ratio (Fig. 8), the optimal sensitivity estimate indicates a marginal increase in $\mathrm{DOC}^{*}$ as this constraint is relaxed below 0.6 when in fact such a decrease results in an infeasible design. Note that the modest slope of the optimal sensitivity prediction shown in Fig. 8 should be expected from the small relative magnitude of the $c \lambda *$ value listed in Table 2 . Figure 9 shows that as the configuration becomes more unstable, the static margin constraint eventually becomes inactive leading to an erroneous prediction of the optimum DOC. This figure also shows that changes in the active set will begin to induce error for an overly stable design.

Figures 5-9 demonstrate that post-optimality information is useful in predicting the effect of various constraint perturbations on DOC*. To illustrate the validity of postoptimality information with respect to a parameter variation, the effects of perturbations in the cruise Mach number were investigated. Fig. 10 shows the variation in optimum DOC

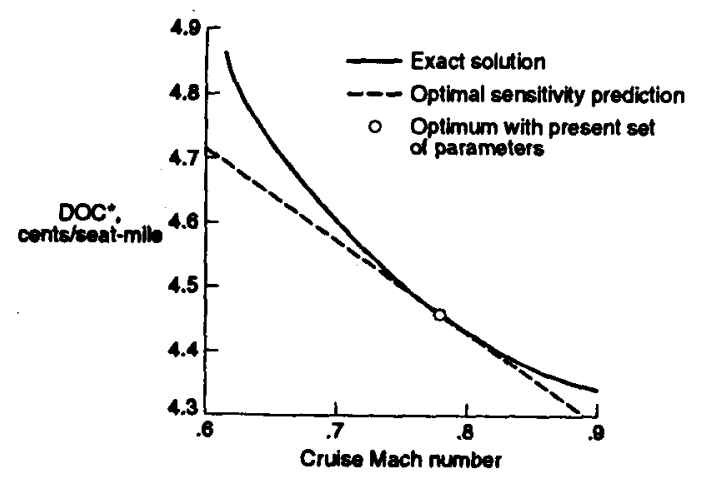

Fig. 10 Comparison of optimal sensitivity prediction for cruise Mach number parameter variations. for different values of the cruise Mach number. Through a central-difference approximation, the slope of the re-optimization curve in Fig. 10 is computed as -1.450 cents/seat-mile at the present optimum; whereas, with use of eq. (1), the change in optimum $D O C$ with respect to cruise Mach number is predicted to be -1.448 cents/seat-mile. Hence, eq. (1), may be used to obtain accurate estimates of the effect of parameter variations on the optimum solution. Fig. 10 shows that for cruise Mach numbers from 0.7 to 0.85 , the post-optimality prediction is quite accurate (to within $1 \%$ of the true value).

Table 3 lists the error in the optimal sensitivity prediction relative to a re-optimized solution for various levels of cruise range constraint perturbations (Fig. 5). Notice that the error is quite small over a large range of perturbations. Also listed in this table is the number of function evaluations required by the NPSOL re-optimization procedure beginning at the present optimum (Fig. 4). Note that in this re-optimization process, no information from the original optimization procedure was retained. Table 3 highlights the payoff in using optimal sensitivity results to predict the effect of small changes in a parameter or constraint. Recall that this estimate is essentially free for constraint variations and costs only one or two extra function evaluations for every parameter of interest. Hence, use of optimal sensitivity information may be much more efficient than re-optimization.

Table 3. Optimal sensitivity estimate versus NPSOL reoptimization for cruise range constraint variation.

\begin{tabular}{|c|c|c|c|c|}
\hline \multicolumn{5}{|c|}{$\begin{array}{l}\text { Constraint originally }=960 \mathrm{n} \cdot \mathrm{mi} \\
\begin{aligned} D O C^{*} & =4.4594 \text { cents } / \text { seat-mile } \\
\lambda^{*} & =-9.7517 \theta-04\end{aligned}\end{array}$} \\
\hline $\begin{array}{c}\text { Constraint } \\
\text { Bound, } \\
\text { n.mi. }\end{array}$ & $\begin{array}{c}\text { Actual DOC: } \\
\text { cents/seat-mile }\end{array}$ & $\begin{array}{l}\text { Predicled DOC: } \\
\text { cents/seat-mile }\end{array}$ & $\begin{array}{l}\% \text { Error } \\
\text { in DOC* }\end{array}$ & $\begin{array}{c}\text { Function Evaluations } \\
\text { Required by } \\
\text { Re-optimization } 1\end{array}$ \\
\hline $\begin{array}{r}500 \\
600 \\
700 \\
850 \\
900 \\
925 \\
950 \\
955 \\
960 \\
965 \\
970 \\
1000 \\
1100 \\
1250 \\
1500 \\
2000\end{array}$ & $\begin{array}{l}5.3930 \\
5.0649 \\
4.8331 \\
4.5850 \\
4.5235 \\
4.4956 \\
4.4695 \\
4.4643 \\
4.4594 \\
4.4545 \\
4.4496 \\
4.4218 \\
4.3451 \\
4.2552 \\
4.1728 \\
4.1198\end{array}$ & $\begin{array}{c}4.9080 \\
4.8105 \\
4.7129 \\
4.5667 \\
4.5179 \\
4.4935 \\
4.4692 \\
4.4643 \\
- \\
4.4545 \\
4.4496 \\
4.4204 \\
4.3232 \\
4.1766 \\
3.9330 \\
3.4451\end{array}$ & $\begin{array}{l}9.0 \\
5.0 \\
2.5 \\
0.4 \\
0.1 \\
0.05 \\
0.01 \\
0 \\
- \\
0 \\
0 \\
0.03 \\
0.50 \\
1.85 \\
5.75 \\
16.40\end{array}$ & $\begin{array}{r}406 \\
469 \\
350 \\
456 \\
400 \\
138 \\
91 \\
91 \\
- \\
62 \\
62 \\
91 \\
155 \\
487 \\
416 \\
555\end{array}$ \\
\hline
\end{tabular}

1Original optimization problem required 610 function evaluations. 
The basic premise behind NPSOL (or any SQP algorithm) involves the use of major and minor iterations.4,12 The major iterations determine the sequence of design points which eventually converge to the solution; hence, it is in each major iteration that the line-search is performed. Minor iterations are used to solve the resulting quadratic programming problem at each design point. Since there are 14 design variables in this problem (including the two compatibility variables), every minor problem iteration will require at least 14 function evaluations to numerically estimate the objective gradient at the present point. Furthermore, because there may be several minor iterations in one major iteration (particularly, during the first major iteration where the algorithm is trying to identify an initial feasible point), even a re-optimization in which only a single major iteration is needed may require a large number of function evaluations. As the perturbation from the original problem increases, so will the number of function evaluations required in the re-optimization process. This increase in numerical requirements must be balanced against the failing accuracy of the optimal sensitivity prediction for large perturbations.

As shown in Table 3, for even a small perturbation in the constraint value, NPSOL re-optimization requires a large number of function evaluations to reach the new optimum. This is because when the value of an active constraint is perturbed, either the present design point (the previous optimum) becomes infeasible or the perturbed constraint becomes inactive. As mentioned previously, either of these two situations generally requires more than one minor iteration (thereby, increasing the number of objective gradient evaluations required for the new solution). In general, by beginning the re-optimization procedure with information built-up during the initial problem solution, the number of function evaluations may be reduced. However, in this case, solution of the perturbed problem may still require several minor iterations to either locate a feasible point or identify the correct active set of constraints. Therefore, starting the re-optimization procedure in this manner was not found to provide a dramatic decrease in the required number of function evaluations.

Use of Optimal Sensitivity Information in Hierarchical Decomposition

Numerous authors have proposed the use of some form of multi-level decomposition strategy to simplify the solution of a complex design problem. ${ }^{17-20}$ In such an approach, a single, large problem is decomposed into several smaller problems, each of which is optimized separately. Although the solution of the decomposed problem now requires an optimization routine for each smaller problem, the smaller problems are simpler to analyze and properly scale. When broken down into master and subproblems, the decomposition is termed hierarchical. ${ }^{18,19}$

From the previous analysis, it is evident that if either a constraint or parameter is perturbed slightly, the sensitivity prediction will yield a good estimate even in a nonlinear region of the design space. Because the optimal sensitivity information is nearly exact in the limit of an infinitesimally small step away from the solution, a design problem which is hierarchically decomposed could utilize the post-optimality information of the subproblem to estimate the main problem objective gradient. This is possible since the subproblem parameters are also the main problem design variables. Hence, the main problem objective gradient $(\mathrm{dF} / \mathrm{dx})$ is equivalent to the subproblem $\mathrm{dF}^{*} / \mathrm{dp}$ and can be estimated with eq. (1) rather than through finite differentiation of the reoptimized subproblem.

This use of sensitivity information is demonstrated through the design of a fully-reusable, single-stage-to-orbit (SSTO) vehicle. Dry-weight is the minimization variable since for a manned launch vehicle, the dry-weight components comprise a major portion of the total development cost. This problem has been analyzed previously through use of a Taguchi approach in which a form of hierarchical decomposition was used. 21,22 Additionally, this problem has been treated with and without decomposition using calculus-based optimization. ${ }^{23}$ In this investigation, the problem is posed with 25 design variables, and five constraints. As listed in Table 4, there are three terminal constraints in addition to a maximum dynamic pressure and maximum normal force constraint. Design variables include the gross weight and thrust-to-weight ratio at liftoff, the initial launch direction, the reference aerodynamic surface area, six propulsion parameters, and a set of 15 ascent pitch rates.

To analyze this problem, the three degree-of-freedom equations of motion were numerically integrated with use of the Program to Optimize Simulated Trajectories. ${ }^{24}$ As shown in Fig. 11, the required set of POST inputs include all 25 design variables. POST is used to evaluate the inflight and terminal constraints and to compute the vehicle massratio (MR). For a given MR, the Configuration Sizing program developed at LaRC is used to size the vehicle and determine the dry-weight. As shown in Fig. 11, the six propulsion parameters and the liftoff thrust-to-weight are the only design variables required by CONSIZ. 
Table 4. Single-stage-to-orbit launch vehicle design problem.

\section{Objective function $=$ dry woight}

\section{Constraints}

\begin{tabular}{|c|c|}
\hline Name & Required Value \\
\hline \multicolumn{2}{|c|}{ 1. Terminal altitude, ft ....................................................3.0380505 } \\
\hline \multicolumn{2}{|c|}{ 2. Terminal tlight path angle, deg .....................................0.0 } \\
\hline \multicolumn{2}{|c|}{ 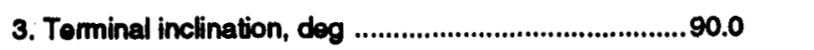 } \\
\hline \multicolumn{2}{|c|}{ 4. Maximum dynamic pressure, $1 \mathrm{~b} / \mathrm{tt}^{2} \ldots \ldots \ldots \ldots \ldots \ldots . . . . . . . . .1000 .0$} \\
\hline 5. Maximum normal & ..........3.65e5 \\
\hline
\end{tabular}

Design Variables

\begin{tabular}{|c|c|c|c|}
\hline & Name & Initial Value & Max. \\
\hline 1. & Gross liftoff woight, Ib ............1.006 & 2.006 & 2.006 \\
\hline 2. & $S_{\text {rel, wing, }} f^{2} \ldots \ldots \ldots \ldots \ldots . . \ldots 1000.0$ & 1500.0 & 2000.0 \\
\hline 3. & Launch azimuth, deg ..............160.0 & 180.0 & 200.0 \\
\hline 4. & Thrust/woight at liftoff ................. 1.2 & 1.35 & 1.5 \\
\hline 5. & Chamber pressure, psia ....... 3000.0 & 3850.0 & 4700.0 \\
\hline 6. & Mode 1, mixture ratio ................ 10.0 & 12.0 & 14.0 \\
\hline 7. & Mode 2, mixture ratio ................. 5.0 & 6.0 & 7.0 \\
\hline 8. & Mode 1, area ratio .....................20.0 & 40.0 & 60.0 \\
\hline 9. & Mode 2, area ratio .....................60.0 & 110.0 & 160.0 \\
\hline 10. & $\begin{array}{l}\text { Mode } 1 / \text { mode } 2 \text { transition } \\
\text { Mach number ............................... } 1.5\end{array}$ & 3.0 & 4.5 \\
\hline $11-25$ & Set of 15 pitch rates, deg/sec. .0 .0 & & -120.0 \\
\hline
\end{tabular}

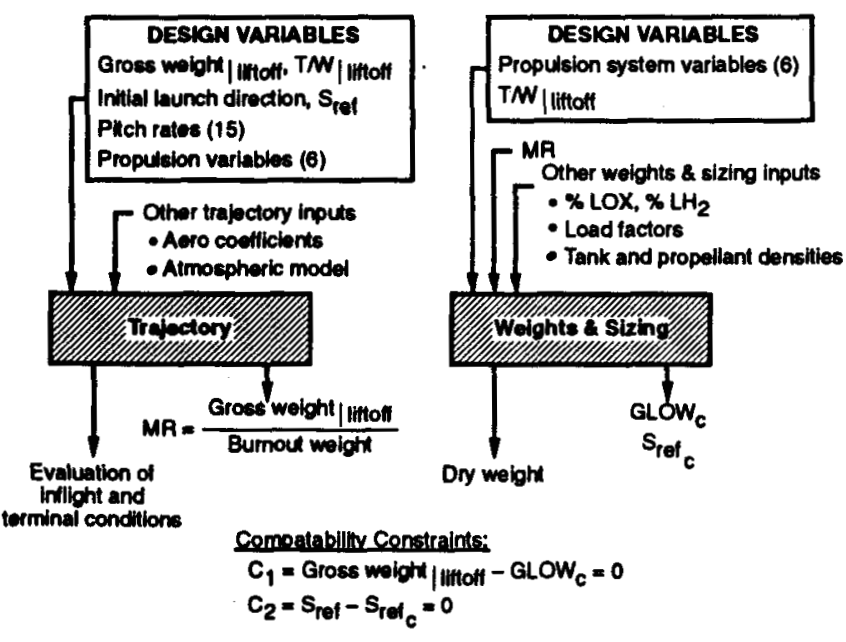

Fig. 11 Trajectory/weights and sizing integration process.

As part of the sizing process, CONSIZ scales the vehicle and re-computes the gross liftoff weight $\left(\mathrm{GLOW}_{\mathrm{c}}\right)$ and reference aerodynamic surface area $\left(\right.$ Sref $_{\mathcal{c}}$ ). Therefore, configuration-control is required between POST and CONSIZ to ensure that the reference aerodynamic surface area re- sulting from the vehicle sizing process (Srefc) is the same as the reference aerodynamic surface area used to compute the aerodynamic forces and evaluate the flight path (Sref). Similar control must be placed on the gross liftoff weight. These requirements could either be enforced by iteration or through the addition of two extra constraints (compatibility constraints) as shown in Fig. 11. By using compatibility constraints, the configuration-control requirements are placed on the optimizer and an iterative loop is removed.

Removal of the POST-CONSIZ iteration loop reduces the required number of function evaluations. Additionally, integration of POST and CONSIZ in this manner has numerous design benefits. In regards to optimization, integrating these two codes allows dry-weight or other vehicle component weights to become available as optimization variables. Furthermore, through integration, the combined vehicle-trajectory model is guaranteed to be consistent. Note that prior to the integration of these two disciplinary algorithms, a designer was forced to iterate between these two codes acting as a human interface.

Before resorting to decomposition, the solution of this SSTO optimization problem was attempted with use of a single optimizer as shown in Fig. 12. Use of both NPSOL ${ }^{12}$ and a projected-gradient approach were tried; however, a converged solution was never achieved. Similar problems with a single optimizer were encountered in Ref. 23. It is believed that most of this convergence difficulty may be attributed to scaling problems. With use of a hierarchical decomposition approach, as sketched in Fig. 13, convergence was shown to be much easier to achieve. This is most likely a result of two factors. First, when decomposed, the number of optimization degrees-of-freedom (defined as the number of design variables minus the number of active constraints at the solution) in each of the two optimization problems is less than in the original problem. For this problem, when decomposed, the subproblem is characterized with 12 optimization DOF and the main problem with seven optimiza-

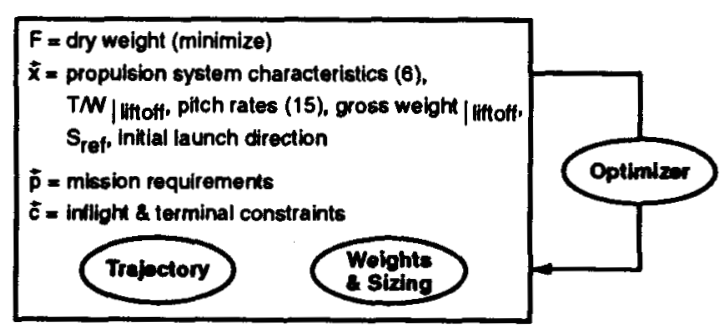

Fig. 12 SSTO launch vehicle design problem, single optimization. 


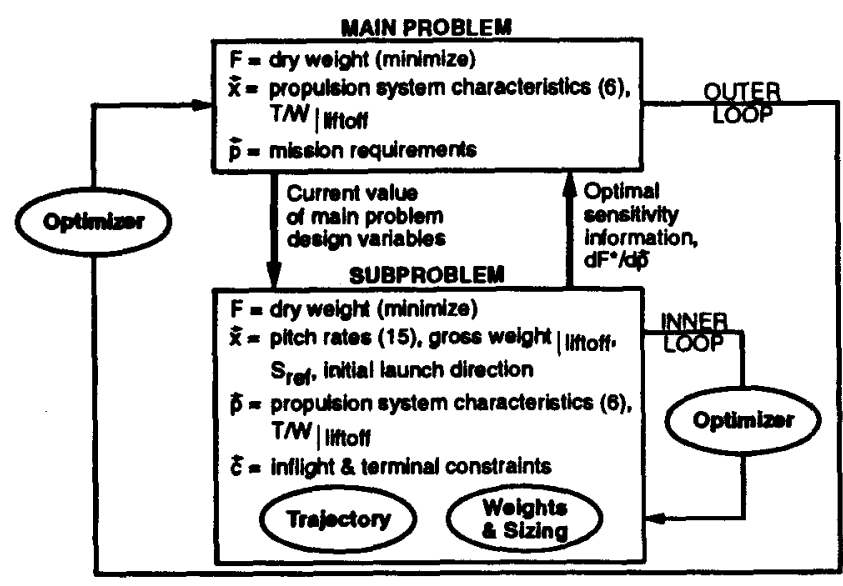

Fig. 13 SSTO launch vehicle design problem, hierarchical decomposition optimization.

tion DOF; whereas the original problem has 19 optimization DOF. Less optimization DOF typically implies less difficulty for the optimizer once a feasible point has been obtained. Secondly, by decomposing the problem such that the trajectory variables are in the subproblem and the propulsion variables are in the main problem (Fig. 13), disciplinary cross-coupling within each problem becomes less of an issue (i.e., the terminal and inflight trajectory constraints are satisifed by variations in the trajectory design variables).

As a result of the decomposed structure of this design problem, computation of the main-problem objective gradient could require a subproblem optimization for each mainproblem variable perturbation. This implies seven subproblem optimizations (each requiring numerous function evaluations) would be needed for every main-problem minor iteration. However, as illustrated in Figure 13, some of this computational expense could be eliminated by passing the optimal sensitivity information of the converged subproblem to the main problem. This is possible since the parameters of the subproblem are the design variables of the main problem. In this case, only seven extra function evaluations are required to estimate the main-problem objective gradient through eq. (1).

Convergence results and the optimum dry-weight for several optimization strategies are presented in Table 5 along with the prior solution of Refs. 21 and 22 . As mentioned previously, with just a single optimizer, converged solutions were not achieved; therefore, for these cases, the final value of dry-weight is above that determined in the earlier studies. However, when decomposed as described in Fig. 13, only 11 main-problem major iterations were required for NPSOL to converge to the optimum solution (beginning from the design point listed in Table 4). Without the use of the subproblem optimal sensitivity information in the main-problem, 477 function evaluations were required to reach this solution.

When the main-problem objective gradient calculations were based on the subproblem optimal sensitivity calculations, the number of function evaluation required to reach the optimum decreased to 349 (a decrease of 27\%). An even greater decrease could have been achieved; however, during the first two main-problem major iterations, finite-differencing was needed to compute the objective gradient. This was required because with the initial set of design variables, all of the inflight and terminal constraints could not be satisfied by the subproblem optimization. With an infeasible result in the subproblem, the computation of $\mathrm{dF}^{*} / \mathrm{dp}$ from eq. (1) could not be performed. Once the subproblem constraints were satisfied, the optimal sensitivity information could be computed and passed from the subproblem to the main problem, thereby avoiding the need for numerous subproblem optimizations to compute the main-problem objective gradient.

The values of the optimum design variables and objective function obtained through solution of the hierarchically decomposed problem which utilized the post-optimality information are shown in Fig. 14 along with a vehicle sketch.

Table 5. Single-stage-to-orbit launch vehicle optimization results.

\begin{tabular}{|l|c|c|c|}
\hline \multicolumn{1}{|c|}{$\begin{array}{c}\text { Optimization } \\
\text { Approach }\end{array}$} & $\begin{array}{c}\text { Trajectory/ } \\
\text { Weights } \\
\text { \& Sizing } \\
\text { Analyses }\end{array}$ & $\begin{array}{c}\text { Required } \\
\text { \# of } \\
\text { Function } \\
\text { Evaluations }\end{array}$ & $\begin{array}{c}\text { Final } \\
\text { Objective } \\
\text { Function } \\
\text { Value, lb }\end{array}$ \\
\hline $\begin{array}{l}\text { Taguchi Method } 2122 \\
\text { Single Optimization } \\
\text { Problem Projected } \\
\text { Gradient Method } \\
\text { Single Optimization } \\
\text { Problem NPSOL } \\
\text { Hierarchical } \\
\text { Decomposition } \\
\text { with Finite } \\
\text { Differencing in Main } \\
\text { Problem NPSOL } \\
\text { Hierarchical } \\
\text { Decomposition } \\
\text { with Post-Optimality } \\
\text { Information } \\
\text { in Main Problem }\end{array}$ & Integrated & $>1000$ & 109,400 \\
\hline
\end{tabular}




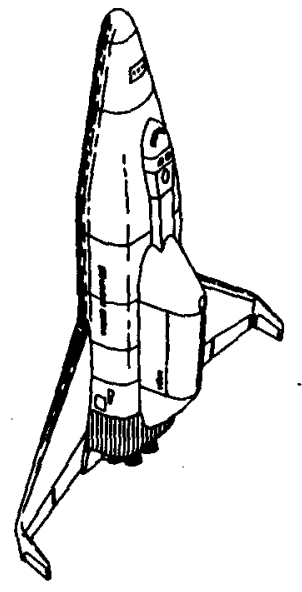

Gross liftoff weight, lb . . . . 1.23906 $s_{\text {ref }} t^{2} \ldots \ldots \ldots \ldots \ldots \ldots 1552$ Launch azimuth, dog $\ldots \ldots .182 .1$ Thrustweight at liftoff . . . . . . 1.2 Chamber pressure, psia . . . . 4700

Mode 1, mixture ratio ........ 10.0

Mode 2, mixture ratio . . . . . . . 7.0

Mode 1, area ratio........ 49.38

Mode 2, area ratio......... 160.0

Mode 1/mode 2 transition

Mach number ........... 1.5

Set of 15 pitch rates

Dry woight, lb 109080

\section{Fig. 14 Optimal solution of single-stage-to-orbit launch vehicle design problem.}

The optimum design variables found matched that of Refs. 21 and 22 quite well. However, because the trajectory and weights and sizing disciplines were integrated, a consistent vehicle-trajectory model results. This consistency resulted in a slightly lower final dry-weight (see Table 5).

\section{Conclusions}

The objective of the present research was to investigate the applicability of optimal sensitivity information to aerospace vehicle design. An optimal sensitivity (or post-optimality) analysis refers to computations performed once the initial optimization problem is solved. These computations may be used to characterize the design space about the present solution and infer changes in the present solution as a result of a constraint or parameter variation without re-optimizing the entire system. This analysis has demonstrated that the post-optimality information generated through first-order computations can be used to accurately predict the effect of constraint and parameter perturbations on the optimal solution. This first-order analysis is essentially free for constraint variations and requires a single extra function evaluation for perturbations in a previously fixed parameter.

For the aircraft design problem investigated, the optimal sensitivity predictions matched the true variation in optimum DOC over the practical range of cruise range, takeoff field length, second-segment climb gradient, drag-to-thrust ratio during cruise, and static margin constraint values to within a few percent. Furthermore, the variation in DOC* with respect to the parameter, cruise Mach number, was also estimated accurately (to within $1 \%$ over the range 0.7 to 0.85 ). Hence, an appropriate use of optimal sensitivity information is to demonstrate the numerous design possibilities available through a change in the constraints or parameters without the need for re-optimization. For even small constraint variations, re-optimization was shown to require a large number of function evaluations because (1) either the previous optimum becomes infeasible or (2) the perturbed constraint becomes inactive. Hence, use of optimal sensitivity information was shown to be much more efficient than re-optimization. However, for large perturbations in a constraint or parameter, active set changes and infeasible regions of the design space cause the optimal sensitivity predictions to become inaccurate and re-optimization is required.

Because the optimal sensitivity information is nearly exact in the limit of an infinitesimally small step away from the solution for parameter variations, a design problem which is hierarchically decomposed could utilize the post-optimality information of the subproblem to estimate the main-problem objective gradient. This is possible since the subproblem parameters are also the main-problem design variables. Estimation of the main-problem objective gradient in this manner, rather than through finite differentiation of the reoptimized subproblem, results in a significant decrease in the number of objective functions required to reach the optimal solution. This use of optimal sensitivity information provided a $27 \%$ decrease in the number of required function evaluations for solution of a reusable, single-stage-to-orbit, launch vehicle design problem.

To solve this launch vehicle design problem, integration of a trajectory program and weights and sizing algorithm was performed. This integration relied on compatibility constraints to enforce configuration-control and eliminate an otherwise costly iteration loop. Through integration of these two disciplinary codes, dry-weight or any other vehicle component weight combination becomes available as a potential optimization variable and a consistent vehicle-trajectory model is ensured. Additionally, this consistency resulted in a slightly lower final objective function value.

\section{References}

1. Sobieski-Sobieszczanski, J., "Sensitivity of Complex, Internally Coupled Systems," ALAA Journal, Vol. 28, No. 1, Jan. 1990.

2. Sobieski-Sobieszczanski, J., "Sensitivity Analysis and Multidisciplinary Optimization for Aircraft Design: Recent Advances and Results," Loumal of Aircraft. Vol. 27, No. 12, Dec. 1990. 
3. Vanderplaats G.N., Numerical Optimization Techniques for Engineering Design: With Applications, McGraw-Hill Inc., 1984.

4. Gill, P.E.; Murray, W.; and Wright, M.H., Practical Optimization, Academic Press, Inc, 1981.

5. Sobieski-Sobieszczanski, J.; Barthelemy, J.; and Riley, K.M., "Sensitivity of Optimum Solutions of Problem Parameters," AIAA Joumal, Vol. 20, No. 9, Sept. 1982.

6. Barthelemy, J.; and Sobieski-Sobieszczanski, J., "Extrapolation of Optimum Design Based on Sensitivity Derivatives, AIAAJoumal, Vol. 21, No. 5, May 1983.

7. Beltracchi, T.J.; and Nguyen, H.N., "Experience with Post Optimality Parameter Sensitivity Analysis in FONSIZE (A Conceptual Sizing and Trajectory Optimization Code for Launch Vehicles)," AIAA 92-4749, Fourth AIAANSAF/NASAOAI Symposium on Multidisciplinary Analysis and Optimization, Cleveland, OH, Sept 1992.

8. Fiacco, A.V., Introduction to Sensitivity and Stability Analysis in Nonlinear Programming, Vol. 165, Mathematics in Science and Engineering Series, Academic Press, 1983.

9. Beltracchi, T.J.; and Gabriele, G.A., "An Investigation of New Methods for Estimating Parameter Sensitivities," NASA CR 4245, August 1989.

10. Beltracchi, T.J., and Gabriele, G.A., "Observations on Extrapolations Using Parameter Sensitivity Derivatives," ASME DE Volume 14, Proceedings of the ASME Design Automation Conference, September 1988.

11. Hallman, W., "Sensitivity Analysis for Trajectory Optimization Problems," AIAA 90-0471, 28th Aerospace Sciences Meeting, January 1990.

12.Gill,P.E;Murray,W.;Saunders,M.A.; andWright,M.H.,'Users Guide for NPSOL (Version 4.0): A Fortran Package for Nonlinear Programming," Technical Report SOL 86-2, Department of Operations Research, Stanford University, Jan. 1986.

13. Kroo, I.M., "An Interactive System for Aircraft Design and Optimization," AIAA 92-1190, AIAA Aerospace Design Conference, Irvine, CA, Feb. 1992.

14. Kroo, I.; and Takai, M., "Aircraft Design Optimization Using a Quasi-Procedural Method and Expert System," AIAA/AHS/
ASEE Aircraft Design, Systems and Operations Meeting, AIAA 88-4428, Atlanta GA, Sept. 1988.

15. Kroo, I.; and Takai, M., "A Quasi-Procedural, Knowledge Based System for Aircraft Design," AIAA/AHS/ASEE Aircraft Design, Systems and Operations Meeting, AIAA 88-4428, Atlanta GA, Sept. 1988.

16. Gage, P.; and Kroo, I., "Development of the QuasiProcedural Method for Use in Aircraft Configuration Optimization," AIAA 92-4693, Fourth AIAAUSAFNASA OAI Symposium on Multidisciplinary Analysis and Optimization, Cleveland, OH, Sept. 1992.

17. Barthelemy, J.; "Engineering Application of Heuristic Multilevel Optimization Methods," NASA CP-3031, Recent Advances in Multidisciplinary Analysis and Optimization Conference, Part 3, p. 1029, Sept. 1988.

18. Sobieski-Sobieszczanski, J.; James, B.B.; and Riley, M.F.; "Structural Sizing by Generalized, Multilevel Optimization," AJAA Joumal, Vol. 25, No. 1, pp. 139-145, January 1987.

19. Beltracchi, T.J.; "Decomposition Approach to Solving the All-Up Trajectory Optimization Problem," Journal of Guidance, Control, and Dynamics, Vol. 15, No. 3, pp. 707n16, May-June 1992.

20. Korngold, J.; Gabriele, G.; Renaud, J.; and Kott, G.; "Application of Multidisciplinary Design Optimization to Electronic Package Design," AIAA 92-4704, Fourth AIAAUSAF/NASA/OAI Symposium on Multidisciplinary Analysis and Optimization, September 21-23, 1992.

21. Stanley, D.O.; Unal, R.; and Joyner, C.R., "Application of Taguchi Methods to Propulsion System Optimization for SSTO Vehicles," Loumal of Spacecraft and Rockets, Vol. 29, No. 4, pp. 453-459, July-Aug. 1992.

22. Unal, R.; Stanley, D.O.; and Joyner, C.R.; "Propulsion System Design Optimization Using Taguchi Methods," IEEE Transactions on Engineering Management, August 1993.

23. Barnes, A.E., "Improved Aerospace Vehicle Design: Integration of POST and CONSIZ," Masters Thesis, George Washington University, Aug., 1993.

24. Brauer, G.L.; Cornick, D.E.; and Stevenson, R; "Capabilities and Applications of the Program to Optimize Simulated Trajectories," NASA CR-2770, February 1977. 